\title{
Perturbation Theory for Periodic Orbits in a Class of Infinite Dimensional Hamiltonian Systems
}

\author{
Claudio Albanese ${ }^{\star}$ and Jürg Fröhlich \\ Institut für Theoretische Physik, ETH-Hönggerberg, CH-8093 Zürich, Switzerland
}

Received December 11, 1990

\begin{abstract}
We consider a class of Hamiltonian systems describing an infinite array of coupled anharmonic oscillators, and we study the bifurcation of periodic orbits off the equilibrium point. The family of orbits we construct can be parametrized by their periods which belong to Cantor sets of large measure containing certain periods of the linearized problem as accumulation points. The infinitely many holes forming a dense set on which the existence of a periodic orbit cannot be proven originate from a dense set of resonances that are present in the system. We also have a result concerning the existence of solutions of arbitrarily large amplitude.
\end{abstract}

\section{Introduction}

1.1. The purpose of this article is to study periodic solutions of infinite-dimensional Hamiltonian systems describing anharmonic oscillators with random spring constants located on the sites of the $v$-dimensional cubic lattice, $\mathbb{Z}^{\nu}$. The equations of motion are

$$
\frac{d^{2}}{d t^{2}} u(x, t)+[(-\Delta+V(x)) u](x, t)+\lambda W(u)(x, t) u(x, t)=0
$$

where $u$ is a real-valued function, $u: \mathbb{Z}^{n} \times \mathbb{R} \rightarrow \mathbb{R}$ and $\Delta$ is the discrete laplacian defined by

$$
(\Delta u)(x, t)=\sum_{|y-x|=1}(u(y, t)-u(x, t)) .
$$

The spring constants $V(x)$ are i.i.d. random variables taking only positive values, with a probability distribution of $V \equiv V(x)$ given e.g. by

$$
d \varrho(V)=N \theta(V) e^{-|V|^{\alpha} / \zeta} d V,
$$

\footnotetext{
* Address after September 1990: Department of Physics, Princeton University, POB 708, Princeton, NJ 08544, USA
} 
where $\alpha>0, \theta(V)=1$ if $V>0$, and $\theta(V)=0$ otherwise, and $N$ is a normalization constant chosen such that $\int d \varrho(V)=1$; or

$$
d \varrho(V)=\frac{1}{\zeta} \chi[0, \zeta](V) d V,
$$

where $\chi_{I}$ is the characteristic function of an interval $I$.

The operator $W$ in (1.1) is defined as the operator of multiplication by

$$
W(u)(x, t)=\sum_{y} W(|y-x|)|u(y, t)|^{2},
$$

where the kernel $W(|y-x|)$ decays exponentially fast, with rate $m_{W}>0$, as $|y-x| \uparrow \infty$. Finally, the constant $\lambda$ in (1.1) is the perturbation parameter that we vary, while a suitable norm of $u(x, t)$ is held fixed.

We prove two results about periodic solutions of (1.1): The first one is a perturbative result, valid for small $\lambda$, and concerns the existence of a bifurcation branch of solutions of (1.1) around the solutions of the linear problem, ((1.1), with $\lambda=0$ ), corresponding to eigenfunctions of $H^{0}=-\Delta+V$ with large eigenvalue. Due to an infinite number of resonances densely distributed around all eigenvalues of $H^{0}$, we construct branches of periodic solutions whose periods do not sweep out intervals, but only Cantor sets of large Lebesgue measure. As a second result, we can prove the existence of certain periodic solutions of Eq. (1.1) for arbitrarily large $\lambda$, using the methods in [AFS]. However, since the extension of the proof of [AFS] requires only some of the ideas used in the proof of the first result of this paper, along with the methods developed in [AFS], we do not present any details of the proof of our second result. We remark that, since the spring constants are random, we are interested in proving statements which are true for all choices of $V(x), x \in \mathbb{Z}^{v}$, in a full-measure set of $V$ 's.

This paper is organized as follows. In the present, introductory section, we review some of the earlier work on problems related to the ones just described, and then we state the two results mentioned above in the form of theorems, (Theorems 1 and 2). In Sect.2, we describe the strategy of the proof of our first result, (Theorem 1). Some details of the proof are deferred to Sect.3. Not all details are explicitly worked out, because some of them are essentially contained in our two previous articles, $[\mathrm{AF}]$ and $[\mathrm{AFS}]$. The results proven in this paper have been announded in $[\mathrm{AF}]$ as part III.

1.2. We consider the random Schrödinger operator $H^{0}=-4+V$, with the parameter $\zeta$ in (1.3) and $\left(1.3^{\prime}\right)$ interpreted as a measure of the disorder in the distribution of $V$. It is known that the part

$$
\operatorname{spec}\left(H^{0}\right) \cap\left[E_{1}(v, \zeta), \infty\right)
$$

of the spectrum of $H^{0}$ is pure-point, consisting of a dense set of simple eigenvalues, and that the constant $E_{1}(v, \zeta) \geq 0$ vanishes for large enough $\zeta$. We shall only consider periodic vibrations of frequency $\omega \equiv \sqrt{E} \geq \sqrt{E_{1}(v, \zeta)}$. The results on random Schrödinger operators needed in our analysis are proven in [FMSS, SW and DLS]. The proofs of [SW and DLS] combine a functional analytic argument with estimates on the decay of Green's functions derived in [FS]. The methods of [FMSS] are more involved, but since they are constructive, they provide some further insights into the problem which are useful in our analysis. 
In [AF and AFS], we study stationary solutions of the non-linear Schrödinger equation

$$
i \frac{d u}{d t}=(-\Delta+V+\lambda W(u)) u,
$$

i.e., periodic solutions of the form $u(x, t)=e^{-i E t} v(x)$, where $v(x)$ solves the non-linear eigenvalue problem (n.l.e.p.)

$$
(-\Delta+V) v+\lambda W(v) v=E v, \quad(v(x) \in \mathbb{R}) .
$$

We impose the normalization condition

$$
\|v\|_{2}^{2}=\sum_{x} v(x)^{2}=1
$$

and let the parameter $\lambda$ vary. In $[\mathrm{AF}]$, we study the problem for small $\lambda$ perturbatively. Namely, we fix an eigenvalue $E_{0}$ of $(-\Delta+V)$, with eigenfunction $v_{0}(x)$, and ask whether the n.l.e.p. (1.7) with $\lambda$ small, has a solution $\left(E_{\lambda}, v_{\lambda}\right)$ close to $\left(E_{0}, v_{0}\right)$. We prove the following result.

Theorem NRS 1. (i) There exist a full-measure set of potentials, $\Omega$, and a constant $E_{2}(v, \zeta) \geq 0$ with $E_{2}(v, \zeta)=0$ for $\zeta$ large, such that if $E_{0} \geq E_{2}(v, \zeta)$ and $V \in \Omega$, then there is a family of eigensolutions, $\left(E_{\lambda}, v_{\lambda}\right)_{\lambda \in \Lambda}$ of (1.7). Here, $\Lambda$ is a (non-empty) set of $\lambda$ 's having the origin as an accumulation point, and we have that

$$
\lim _{\substack{\lambda \rightarrow 0, \lambda \in \Lambda}}\left(E_{\lambda}, u_{\lambda}\right)=\left(E_{0}, u_{0}\right)
$$

"NRS" stands for "Nonlinear Random Schrödinger problem." The methods on which our proof is based do not allow us to study the geometrical properties of the set $\Lambda$. However, we can show that the set

$$
\mathscr{E}=\left\{E_{\lambda}\right\}_{\lambda \in \Lambda}
$$

of non-linear eigenvalues is a Cantor set of large Lebesgue measure. More precisely, we have

Theorem NRS 1. (ii) The set $\mathscr{E}$ is a Cantor set and

$$
2 \varepsilon_{k}-l\left(\mathscr{E} \cap\left[E_{0}-\varepsilon_{k}, E_{0}+\varepsilon_{k}\right]\right)=0\left(\varepsilon_{k}^{p}\right)
$$

for any power $p>0$, where $\varepsilon_{k}$ is some sequence converging to 0 , as $k \rightarrow \infty$. Here, $l$ denotes Lebesgue measure.

The reason why $\mathscr{E}$ is a Cantor set, and not a interval, is that the eigenvalue $E_{0}$ is not isolated but is surrounded by a dense set of other eigenvalues of $-\Delta+V$.

In our joint paper with Thomas Spencer, [AFS], we prove the following result that is complementary to Theorem NRS 1 in several respects.

Theorem NRS 2. There is a constant $E_{3}(v, \zeta) \geq 0$, with $E_{3}(v, \zeta)=0$ for $\zeta$ large, such that the following statement is true: For all $\lambda>0$, there is a full-measure set of potentials, $\Omega(\lambda)$, such that if $V \in \Omega(\lambda)$ the n.l.e.p. (1.7), (1.8) has an infinite number of solutions corresponding to eigenvalues which form a dense subset of the set $\left\{E \geq E_{3}(v, \zeta)\right\}$.

1.3. In this article, we extend the two theorems above to the system of anharmonic oscillators described by Eq. (1.1). Since we are interested in periodic solutions, 
we can restate our problem as a nonlinear eigenvalue problem. We look for a solution $u(x, t)$ of the form

$$
u(x, t)=\left(\left|\partial_{t}^{2}\right|^{-1 / 2} v\right)(x, \omega t),
$$

where $\omega>0$, and $v(x, t)$ is a (real) function in $L^{2}\left(\mathbb{Z}^{v} \times[-\pi, \pi]\right)$ that is odd and periodic in $t$, i.e.,

$$
v(x,-t)=-v(x, t), \quad v(x,-\pi)=v(x, \pi)
$$

and is subject to the normalization condition

$$
\|v\|_{2}^{2} \equiv \int_{-\pi}^{\pi} d t\left(\sum_{x \in \mathbb{Z}^{v}} v(x, t)^{2}\right)=1 .
$$

The operator $\left.\mid \partial_{t}^{2}\right\}$ in (1.12) is the modulus of the second order time derivative $\partial_{t}^{2}$ acting on the subspace of $L^{2}([-\pi, \pi])$ consisting of smooth functions satisfying (1.13). The eigenfunctions of $\left|\partial_{t}^{2}\right|$ are $\sin (n t), n=1,2,3, \ldots$, and the corresponding eigenvalues are $n^{2}$. The ansatz (1.12) is a periodic solution of (1.1) with period $\frac{2 \pi}{\omega}$, provided $v$ solves the equation

$$
\tau[-\Delta+V+\lambda W(\tau v)] \tau v=\omega^{2} v
$$

where

$$
\tau=\left|\partial_{t}^{2}\right|^{-1 / 2}
$$

The basic difference between problem (1.15) and the non-linear Schrödinger equation (1.7) resides in the spectrum of their linear parts. For arbitrary regions, $R$, in the lattice $\mathbb{Z}^{v}$, let us consider the restriction

$$
H_{R}^{0}=(-\Delta+V)_{R}
$$

of $-A+V$ to $l^{2}(R)$ with zero Dirichlet data at the boundary $\partial R$. If $\sigma\left(H_{R}^{0}\right)$ and $\sigma\left(\tau H_{R}^{0} \tau\right)$ denote the spectra of the operators $H_{R}^{0}$ and $\tau H_{R}^{0} \tau$, respectively, we have that

$$
\sigma\left(\tau H_{R}^{0} \tau\right)=\bigcup_{j=1}^{\infty} j^{-2} \sigma\left(H_{R}^{0}\right) .
$$

Here and in the following, we use the convention that if $A \subset \mathbb{R}$ is a set of real numbers and $\alpha \in \mathbb{R}, \alpha \cdot A$ denotes the set $\{\alpha a \mid a \in A\}$. Two eigenvalues $E_{1}$ and $E_{2}$ in $\sigma\left(\tau H_{R}^{0} \tau\right)$ resonate, i.e., are very close, if there are two frequencies $\omega_{1}$ and $\omega_{2}$, with $\omega_{1}^{2}$ and $\omega_{2}^{2}$ in $\sigma\left(H_{R}^{0}\right)$, and two integers $p_{1}, p_{2}$ such that

$$
\sqrt{E_{1}}=\omega_{1} p_{1} \sim \omega_{2} p_{2}=\sqrt{E_{2}} .
$$

In particular, if $\omega^{2} \in \sigma\left(H_{R}^{0}\right)$ then $\omega$ is a resonant principal harmonic if $j^{2} \omega^{2} \in$ $\sigma\left(H_{R}^{0}\right)$, for some $j>1$, or if $\omega^{2}$ is degenerate.

The main theorem we prove here is the following extension of Theorem NRS 1.

Theorem 1. (i) There is a constant $E_{4}(v, \zeta)$ such that for almost every $V$ and for every simple eigenvalue $E_{0}>E_{4}(v, \zeta)$ with the property that $n^{2} E_{0}$ is not an eigenvalue of $-\Delta+V$, for all $n=2,3, \ldots$, there are a set $\Lambda \subset \mathbb{R}$ containing 0 as an 
accumulation point and a family $\left(v_{\lambda}, \omega_{\lambda}\right)_{\lambda \in \Lambda}$ of solutions of (1.13), (1.14) and (1.15), such that

$$
\lim _{\substack{\lambda \rightarrow 0 \\ \lambda \in \Lambda}}\left\|v_{\lambda}-v_{0}\right\|_{2}=0
$$

where $v_{0}(x, t)=u_{0}(x) \sin t$ and $u_{0}$ is an eigenfunction of $-\Delta+V$ with eigenvalue $E_{0}=\omega_{0}^{2}$. If $\zeta$ is large enough, we have that $E_{4}(v, \zeta)=0$.

(ii) The set $\mathcal{O}=\left\{\omega_{\lambda}\right\}_{\lambda \in \Lambda}$ is a Cantor set, and there exists a sequence $\varepsilon_{k} \downarrow 0$, as $k \uparrow \infty$, such that

$$
2 \varepsilon_{k}-l\left(\mathcal{O} \cap\left[\omega_{0}-\varepsilon_{k}, \omega_{0}+\varepsilon_{k}\right]\right)=0\left(\varepsilon_{k}^{p}\right)
$$

for all powers $p<\infty$.

Remark 1. The assumption that $E_{0}$ is a simple eigenvalue such that the points $n^{2} E_{0}$ are not eigenvalues, for all $n=2,3, \ldots$, is probably not necessary. In fact, we expect that these conditions are fulfilled with probability one. They are known to hold for $d \varrho$ as in $\left(1.3^{\prime}\right)$ and $E_{0}$ large enough.

Remark 2. Walter Craig and C. Eugene Wayne have announced to have proven a result very similar to Theorem 1 for the wave equation

$$
\left(\partial_{t}^{2}-\partial_{x}^{2}+V(x)\right) u(x, t)+W(u)(x, t)=0,
$$

where $u: S^{1} \times \mathbb{R} \rightarrow \mathbb{R}, V(x)$ is a potential on the circle $S^{1}$ satisfying suitable nonresonance conditions, and $W(u)(x, t)$ is a nonlinear term. Their method appears to be rather different from ours.

Finally, let us state the extension of Theorem NRS 2 holding for Eq. (1.1).

Theorem 2. There is a constant $E_{5}(v, \zeta) \geq 0$, with $E_{5}(v, \zeta)=0$, for $\zeta$ large enough, such that the following is true: For all $\lambda>0$, there exists a full-measure set $\Omega(\lambda)$ of potentials such that if $V \in \Omega(\lambda)$ then $E q$. (1.1) has an infinite number of periodic solutions, $u(x, t)$, with

$$
\left\|\left|\partial_{t}^{2}\right|^{1 / 2} u\right\|_{2}=\sqrt{\lambda},
$$

the frequencies of which form a dense subset of $\left\{\omega: \omega^{2}>E_{5}(v, \zeta)\right\}$.

\section{Strategy of the Proof of Theorem 1}

In this section we describe our strategy for the proof of Theorem 1. We replace the original lattice $\mathbb{Z}^{v}$ by an arbitrarily large but finite subset, $L$, of $\mathbb{Z}^{v}$. By $-\Delta$ we mean the finite-difference Laplacian on $l^{2}(L)$ with zero Dirichlet data at $\partial L$. We shall prove estimates that are uniform in $L$, so that, in the end, we can pass to the limit $L \uparrow \mathbb{Z}^{v}$.

Following $[\mathrm{AF}]$, we consider the eigenvalue problems

with

$$
\tau\left[-\Delta+V+(\lambda+i \delta) W\left(\left|\tau v_{\lambda \delta}\right|\right)\right] \tau v_{\lambda \delta}=E_{\lambda \delta} v_{\lambda \delta}
$$

$$
\int_{-\pi}^{\pi} d t\left(\sum_{x \in L}\left|v_{\lambda \delta}(x, t)\right|^{2}\right)=1,
$$

where $\lambda$ are $\delta$ and real parameters. Let $E_{0}=\omega_{0}^{2}$ be an eigenvalue of

$$
-\Delta+V
$$


with $E_{0}>E_{1}(\zeta)$, where $E_{1}(\zeta)$ is the constant appearing in (1.5). Our aim is to construct an open set $P\left(E_{0}, V\right) \subset \mathbb{C}$, and a smooth family $\left(v_{1, \delta}, E_{\lambda, \delta}\right)$ of solutions of $(2.1)$ parametrized by $(\lambda, \delta)$ with

$$
\lambda+i \delta \in P\left(E_{0}, V\right) .
$$

The first step to take is to locate the eigenfunction $u_{0}(x)$ of $-\Delta+V$ in the lattice $L$. Following $[\mathrm{AF}]$, let us define cubes $\tilde{\Lambda}_{j}, \Lambda$, such that

$$
\tilde{\Lambda}_{j}=\left\{x:|x| \leq d_{j}\right\}, \quad \Lambda_{j}=\left\{x:|x| \leq 4 d_{j}\right\},
$$

where $|x|=\max _{1 \leq x \leq v}\left|x^{\alpha}\right|$, and

$$
d_{j}=\exp \left(\beta(5 / 4)^{j}\right)
$$

for $j=1,2, \ldots$, where $\beta>0$ is a constant to be fixed in the following. Let $A_{j}$ be the annulus $\Lambda_{j+1} \backslash \tilde{\Lambda}_{j}$. By using the decay estimates on Green's functions contained in [FMSS], we prove, in Sect. 3 , that there is an integer $\vec{k}$, depending on $V$ and on $E_{0}$, such that the following statements hold true:

(i) The eigenfunction $u_{0}$ of $H^{0} \equiv-\Delta+V$ has uniform exponential decay outside $\Lambda_{\bar{k}}$, i.e.,

$$
\left|u_{0}(x)\right| \leq \exp \left(-m\left(E_{0}\right)|x|\right), \quad x \notin \Lambda_{\bar{k}},
$$

where $m\left(E_{0}\right) \sim \ln E_{0}$, as $E_{0} \uparrow \propto$.

(ii) Eigenvalues, $E$, of $H^{0}$ corresponding to eigenfunctions, $u$, localized outside $\Lambda_{\bar{k}}$, have the following property: If $\left|n^{-2} E-E_{0}\right| \leq e^{-\sqrt{d_{j}}}$, for some $n=1,2, \ldots$, and some $j>\bar{k}$, then $u$ is localized outside $\tilde{\Lambda}_{j}$, i.e., the region where $u$ is localized is separated from the region where $u_{0}$ is localized by a distance $z d_{j}-4 d_{\bar{k}} \approx d_{j}$, (for $\beta$ sufficiently large).

Due to the large separation between the localization regions of $u_{0}$ and of those eigenfunctions corresponding to eigenvalues, $E$, such that $n^{-2} E$ is very close to $E_{0}$, for some $n=1,2, \ldots$, resonances arising in this way, after the non-linear term is introduced, can be circumvented by letting the coupling constant $\lambda+i \delta$ make excursions into the complex plane. Resonances associated with eigenvalues corresponding to eigenfunctions localized close to $u_{0}$ cannot be circumvented by excursions of $\lambda+i \delta$ into the complex plane. However, such eigenvalues are finite in number, and, therefore, $E_{0}$ is separated from them with probability one (w.p.1). In the remainder of this section, we present some details concerning the construction of solutions to (2.1).

Let us consider a solution, $v_{i \delta}$, of the nonlinear eigenvalue problem (2.1) bifurcating off an eigenfunction $u_{0}$ of the corresponding linear problem with eigenvalue $E_{0}$. The function $v_{i \delta}$ remains in the set

$$
\begin{aligned}
\mathscr{V}_{\bar{k}} & =\left\{v(x, t) \in l^{2}\left(\mathbb{Z}^{\prime}\right) \otimes L^{2}(-\pi, \pi): \int_{-\pi}^{\pi} d t \max _{x \in \mathbb{Z}^{\prime}}\left|v(x, t)-u_{0}(x) \sin t\right|^{2} \leq \varepsilon_{0},\right. \\
& \text { and } \left.\int_{-\pi}^{\pi} d t|v(x, t)|^{2} \leq e^{-M|x|}, \quad \forall x \notin \Lambda_{\bar{k}}\right\},
\end{aligned}
$$

where $\varepsilon_{0}$ is a constant chosen sufficiently small, and

$$
M=\min \left\{\frac{1}{2} m\left(E_{0}\right), \frac{1}{2} m_{W}\right\},
$$

with $m_{W}$ the decay rate of $W(|y-x|)$. 
Fig. 1
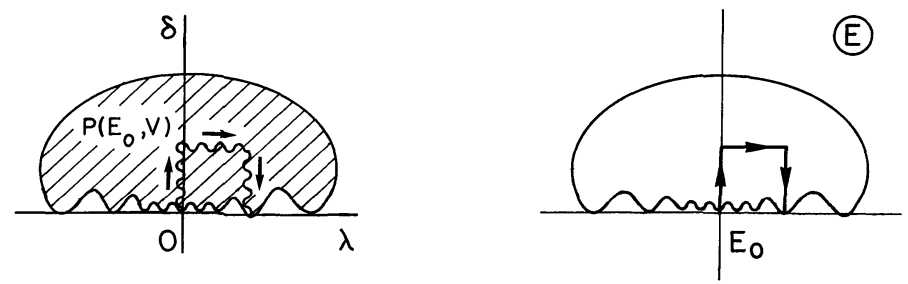

The boundary of $P\left(E_{0}, V\right)$ intersects the real axis on a set $\Lambda$ with the property that, for all $\lambda \in \Lambda$, the limits

and

$$
\lim _{\delta \downarrow 0} u_{\lambda(\delta) \delta} \equiv u_{\lambda}
$$

$$
\lim _{\delta \downarrow 0} E_{\lambda(\delta) \delta} \equiv E_{\lambda},
$$

exist; $(\lambda(\delta), \delta)$ is a path inside $P\left(E_{0}, V\right)$, with the property that $\lim _{\delta \downarrow 0} \lambda(\delta)=\lambda$, which is determined by the condition that $\mathfrak{R} E_{\lambda(\delta) \delta}$ stays constant. The set $\mathcal{O}=\left\{\sqrt{E_{\lambda}}\right\}_{\lambda \in A}$ of frequencies of the solutions we can construct is a Cantor set of large Lebesgue measure, as described in the statement of Theorem 1.

As in [AF], we construct solutions of (2.1) along paths $\gamma_{1} \cup \gamma_{2} \cup \gamma_{3}$ contained in $P\left(E_{0}, V\right)$ such that $E_{\lambda \delta}$ has piecewise constant real or imaginary parts; cf. Fig. 1. On the one hand, one must impose an upper bound on the length of the curve $\gamma_{1} \cup \gamma_{2} \cup \gamma_{3}$ which depends on $V$ and on the spread of the eigenfunction $u_{0}(x)$ that we perturb about. On the other hand, we require a lower bound on the height of the horizontal portion of the curve in the complex E-plane; cf. Fig. 1. However, the closer the path $\gamma_{1} \cup \gamma_{2} \cup \gamma_{3}$ is to the origin, the smaller is that lower bound, and it turns out that the two conditions are compatible, provided the diameter of $P\left(E_{0}, V\right)$ is small enough, for all functions $V$ in a full-measure set and for all sufficiently large eigenvalues $E_{0}$.

The set $\mathscr{E}=\left\{E_{\lambda}\right\}_{\lambda \in \Lambda}$ of positive, real non-linear eigenvalues that can be reached has the form

$$
\mathscr{E}=\mathscr{G}(\bar{k}, V) \cap\left[E_{0}-\varepsilon, E_{0}+\varepsilon\right],
$$

where $\varepsilon$ is a small positive number depending on $V$ and $E_{0}$, and the set $\mathscr{G}(\bar{k}, V)$, called "gap set," is defined as follows:

$$
\begin{aligned}
\mathscr{G}(\bar{k}, V):= & \left\{E \in \mathbb{R}: E>E_{4}(\zeta), \text { and } \forall C \in \mathscr{C}, \forall k^{\prime} \geq \bar{k}, \forall j \in \mathbb{N},\right. \\
& \text { we have that } \operatorname{dist}\left(\sigma\left(H_{C \cap A_{k^{\prime}}}\right), j^{2} E\right) \geq 2 j^{2} e^{\left.-d_{k^{\prime}-1}^{\gamma}\right\}} .
\end{aligned}
$$

Here

$$
\mathscr{C}:=\bigcup_{m=1}^{\infty} \mathscr{C}_{m},
$$

where $\mathscr{C}_{m}$ is the collection of all lattice cubes with faces parallel to lattice planes and edges of length $2^{m}$ which are centered at the sites of $2^{m-1} \mathbb{Z}^{v}$. Moreover, $\bar{k}$ is a sufficiently large integer, depending on $E_{0}$ and on $V$, that will be specified in Lemma 3.4. Finally, $\gamma$ is a positive constant.

For each point $E \in \mathscr{E}$, we can construct solutions $v_{\lambda \delta}$ of $(2.1)$, for all $(\lambda, \delta)$ on a path $\gamma_{1} \cup \gamma_{2} \cup \gamma_{3}$ described in Fig. 1, with

$$
\lim _{\substack{\delta \downarrow 0 \\(\lambda, \delta) \in \gamma_{3}}} E_{\lambda \delta}=E
$$


The eigenvalues $E_{i, \delta}$ of these solutions are simple eigenvalues of the operator

$$
\tau\left[-\Delta+V+(\hat{\lambda}+i \delta) W\left(\left|\tau v_{, \delta}\right|\right)\right] \tau .
$$

Since the non-linear term is small, in an appropriate sense, there is an open neighbourhood around each point $(\lambda, \delta) \in \gamma_{1} \cup \gamma_{2} \cup \gamma_{3}$ in which we can still find a solution of (2.1). The union of such neighbourhoods is the set $P\left(E_{0}, V\right)$ mentioned above.

There are several equivalent ways of controlling the deformation of eigenfunctions along paths like those in Fig. 1. We choose the one that allows for the simplest treatment (known to us) of the small divisor problems that arise in such constructions. Our method is based on the integration of certain differential equations that can be derived as follows: Let $\left(\lambda_{0}, \delta_{0}\right)$ be a point of $P\left(E_{0}, V\right)$, and let $U$ be a small neighbourhood of $\left(\hat{\lambda}_{0}, \delta_{0}\right)$ contained in $P\left(E_{0}, V\right)$. If $\left(\hat{\lambda}_{,} \delta\right) \in U$, then $\left(v_{\lambda \delta}, E_{\lambda \delta}\right)$ solves (2.1) if and only if $v_{\lambda \delta}$ is a fixed point of the nonlinear map.

$$
T^{\lambda \delta}(v)=\frac{1}{2 \pi i} c(v ; \lambda, \delta) \oint_{\Gamma} d z[z-\tau(-\Delta+V+(\lambda+i \delta) W(|\tau v|)) \tau]^{-1} v_{\Lambda_{0} \delta_{0}},
$$

where $c(v ; \lambda, \delta)$ is a normalization factor, $\Gamma$ is a small circle in the complex $E$-plane enclosing $E_{\lambda_{0} \delta_{0}} ; T^{i \delta}(v)$ is defined for $v$ in a small neighbourhood, $N$, of $v_{\hat{\lambda}_{0} \delta_{0}}$ on the unit sphere,

$$
S:=\left\{v \in l^{2}\left(\mathbb{Z}^{v}\right) \otimes L^{2}(-\pi, \pi):\|v\|_{2}=1\right\} .
$$

Since $E_{\lambda_{0} \delta_{0}}$ is a simple eigenvalue of $(2.15)$, for $(\lambda, \delta)=\left(\lambda_{0}, \delta_{0}\right)$, we can choose $\Gamma$, $N$ and $U$ so small that $\Gamma$ encloses one and only one eigenvalue of

$$
\tau[-\Delta+V+(\lambda+i \delta) W(|\tau v|)] \tau,
$$

for all $v \in N$ and all $(\lambda+i \delta) \in U$. By differentiating the fixed point 'equation

$$
v_{i \delta}=T^{i \delta}\left(v_{i \delta}\right)
$$

with respect to $\delta$, we find the differential equation

$$
\begin{aligned}
\frac{\partial v_{\lambda \delta}}{\partial \delta}= & T_{, \delta}^{\lambda \delta}\left(v_{i \delta}\right)+D T^{\lambda \delta}\left(v_{i \delta}\right)\left[\frac{\partial v_{i \delta}}{\partial \delta}\right] \\
= & i Q K^{i \delta}\left(E_{, \delta}\right) \tau W\left(\left|\tau v_{i \delta \delta}\right|\right) \tau v_{\lambda \delta} \\
& +(\lambda+i \delta) Q K^{\lambda \delta}\left(E_{i \delta}\right) \tau D W\left(\left|\tau v_{; \delta}\right|\right)\left[\frac{\partial v_{\lambda \delta}}{\partial \delta}\right] \tau v_{j \delta},
\end{aligned}
$$

where

with

$$
Q=1-\frac{1}{2} P_{v ; \delta}^{0}-\frac{1}{2} \tilde{P}_{v, \delta}^{0}
$$

$$
P_{u}^{0} v=(u, v) u, \quad \tilde{P}_{u}^{0} v=(v, u) u
$$

and

$$
(u, v)=\int_{-\pi}^{\pi}\left[\sum_{x \in \mathbb{Z}^{\prime}} \overline{u(x, t)} v(x, t)\right] d t .
$$

The operator $Q$ arises from the differentiation of the normalization constant, $c$. The operator $K^{i \delta}\left(E_{j \delta}\right)$ is called the "pole-subtracted Green's function" and is defined as the analytic continuation to $z=E_{i, \delta}$ of the operator

$$
K^{\prime \delta}(z)=\left[z-\tau\left(-\Delta+V+(\lambda+i \delta) W\left(\left|\tau v_{, \delta}\right|\right)\right) \tau\right]^{-1}-\left(z-E_{\lambda \delta}\right)^{-1} P_{v, \rho}^{0} .
$$


Finally, $D W$ denotes the ( $\mathbb{R}$-linear!) derivative

$$
D W(|u|)[v]=\frac{\partial W}{\partial \mathfrak{R} u}[\mathfrak{R} v]+\frac{\partial W}{\partial \mathfrak{I} u}[\mathfrak{I} v] .
$$

We refer the reader to the Appendix of $[\mathrm{AF}]$ for a detailed derivation of $(2.20)$ in a similar case.

In order to control the solutions of the deformation equations (2.20), we need to have suitable bounds on the pole-subtracted Green's functions $K^{\lambda \delta}\left(E_{\lambda \delta}\right)$. In [AF], we find bounds valid under the conditions that $v_{\lambda \delta} \in \mathscr{V}_{\bar{k}},|\lambda+i \delta|$ small enough,

$$
\mathfrak{R} E_{\lambda \delta} \in\left(E_{0}-\frac{1}{2} e^{-\sqrt{d_{\bar{k}-1}}}, E_{0}+\frac{1}{2} e^{-\sqrt{d_{\bar{k}-1}}}\right),
$$

and either

$$
\mathfrak{I} E_{\lambda \delta} \geq e^{-\sqrt{d_{\bar{k}}}}
$$

or

$$
\Re E_{\lambda \delta} \in \mathscr{G}(\bar{k}, V),
$$

where $\mathscr{G}(\bar{k}, V)$ is the gap set encountered in (2.12); see also Sect.3. Roughly speaking, $\mathscr{G}(\bar{k}, V)$ is obtained by excising from the set $\left\{E: E>E_{4}(v, \zeta)\right\}$ of energies a small interval around every eigenvalue of $\tau(-\Delta+V) \tau$; see $(2.12)$ and Sect. 3. The result is a Cantor set of fairly large Lebesgue measure. The idea is that, on one hand, when we integrate Eq. (2.20) along the horizontal portion, $\gamma_{2}$, of the path in Fig. 1, the condition (2.27) is fulfilled. On the other hand, if we choose the paths $\gamma_{1}$ and $\gamma_{3}$ in such a way that $\mathfrak{R} E_{\lambda \delta}$ remains constant and belongs to $\mathscr{G}(\bar{k}, V)$, condition (2.28) holds on $\gamma_{1}$ and $\gamma_{3}$. More precisely, on $\gamma_{1}$ and $\gamma_{3}$, we integrate the following system of differential equations

$$
\begin{gathered}
\frac{d}{d \delta} v_{\lambda(\delta) \delta}=\left[1-(\lambda(\delta)+i \delta) Q K \tau D W[\cdot] \tau v_{\lambda(\delta) \delta}\right]^{-1} Q K\left(i+\frac{d \lambda}{d \delta}\right) \tau W \tau v_{\lambda(\delta) \delta}, \\
\frac{d}{d \delta} \mathfrak{R} E_{\lambda(\delta) \delta}=0 .
\end{gathered}
$$

In this paper we give a full discussion of only one aspect of our construction, namely the probabilistic estimates needed to localize an eigenfunction and to construct the gap set. The probabilistic estimates we need are stronger than those contained in $[\mathrm{AF}]$. The other parts of the proof are quite similar to the arguments in $[\mathrm{AF}]$, and we shall refer the reader to that paper.

\section{Probabilistic Estimates}

In the small divisor estimates that go into the proof of Theorem 1, Sect. 1.3, and other related results (Theorems NRS 1, 2, see [AF], [AFS]) and in the analysis of random Schrödinger operators [FS], one requires the following notion of singular sets: For all potentials $V$ and energies $E$, we define a decreasing family of singular sets

$$
S_{0}(E, V) \supseteq S_{1}(E, V) \supseteq S_{2}(E, V) \supseteq \cdots
$$

as follows: We set

$$
S_{0}(E, V):=\left\{x \in \mathbb{Z}^{v}:\left|V(x)-j^{2} \mathfrak{R} E\right| \leq j^{2}(2 v+2 m(E)) \text {, for some } j \in \mathbb{N}\right\}
$$

where $m(E)$ is a constant, with $m(E) \sim \ln E$, as $E \uparrow \infty$. 
The sets $S_{k}(E, V)$, for $k \geq 1$, are defined inductively:

$$
S_{k+1}(E, V)=S_{k}(E, V) \backslash \bigcup_{\alpha} C_{k}^{\alpha},
$$

where $\left\{C_{k}^{\alpha}\right\}$ is a maximal family of disjoint subsets of $S_{k}(E, V)$ satisfying the following conditions:

\section{Condition $k$}

a) $\operatorname{diam}\left(C_{k}^{\alpha}\right) \leq d_{k}$,

b) $\operatorname{dist}\left(C_{k}^{\alpha}, S_{k} \backslash C_{k}^{\alpha}\right) \geq 2 d_{k}^{5 / 4}=2 d_{k+1}$,

c) $\operatorname{dist}\left(\sigma\left(H_{\bar{C}_{k}^{\alpha}}^{0}\right), j^{2} \mathfrak{R} E\right) \geq 2 j^{2} e^{-\sqrt{d_{k}}}$, for all $j \in \mathbb{N}$.

Here $\bar{C}_{k}^{\alpha}$ is a cube in $\mathscr{C}_{m(k)}$ covering $C_{k}^{\alpha}$ where $m(k)$ is determined by the inequalities

$$
2^{m(k)} \geq 10 d_{k} \geq 2^{m(k)-1},
$$

and the collections $\mathscr{C}_{m}$ of lattice cubes have been defined in Sect. 2, after Eq. (2.13).

In the following, we also need singular sets involving only one higher harmonic of the frequency $\omega=\sqrt{\mathfrak{R} E}$. For $j \in \mathbb{N}$, we define

$$
S_{0}^{(j)}(E, V):=\left\{x \in \mathbb{Z}^{v}:\left|V(x)-j^{2} \mathfrak{R} E\right| \leq j^{2}(2 v+2 m(E))\right\},
$$

and

$$
S_{k}^{(j)}(E, V)=\bigcup_{\substack{k^{\prime} \geq k \\ \alpha \mid C_{k^{\prime}}^{\alpha} \cap S_{0}^{(j)}(E, V) \neq \emptyset}} C_{k^{\prime}}^{\alpha} .
$$

The deterministic decay estimates on Green's functions proven in [FS] can be easily extended to the present case, so we shall omit the proofs.

Definition. $A$ set $R \subset \mathbb{Z}^{v}$ is said $(j, k, E)$-admissible iff

$$
\partial R \cap \bar{C}_{k^{\prime}}^{\alpha}=\emptyset
$$

for all $k^{\prime}=0,1, \ldots, k$, and all $\propto$ such that $C_{k^{\prime}}^{\alpha} \cap S_{0}^{(j)}(E, V) \neq \emptyset$. A set which is $(j, k, E)$-admissible for all $j \in \mathbb{N}$ is simply said to be $(k, E)$-admissible.

We have the following deterministic result.

Theorem [FS]. Let $j$ be a positive integer. If the constant $\beta$ in the definition (2.5) of $\left\{d_{k}\right\}$ is chosen large enough, independently of $j$, and if $S_{k}^{(j)}(E, V) \cap \Lambda=\emptyset$, then there is a constant $m \geq \frac{1}{2} m\left(j^{2} E\right)$, independent of $k$, such that, for all $\varepsilon>0$, we have that

$$
\left|\left(-\Delta+V-j^{2}(E+i \varepsilon)\right)^{-1}(x, y)\right| \leq e^{-m|x-y|}
$$

provided $|x-y| \geq \frac{1}{5} d_{k+1}$. If $S_{k}^{(j)}(E, V) \cap \Lambda=\emptyset$ for all $j \in \mathbb{N}$, we have that, for all $\varepsilon>0$,

$$
\left[\tau\left(-\Delta+V-j^{2}(E+i \varepsilon)\right) \tau\right]^{-1}\left(x, n_{1} ; y, n_{2}\right) \leq n_{1}^{2} e^{-m|x-y|} \delta_{n_{1} n_{2}},
$$

provided $|x-y| \geq \frac{1}{5} d_{k+1}$, where the kernel is expressed in the basis

$$
\{\delta(\cdot-x) \sin (n t)\}
$$

of $l^{2}\left(\mathbb{Z}^{\prime \prime}\right) \otimes L^{2}(-\pi, \pi)$. 
Let us fix an open interval, $I$, contained in $\left\{E>E_{4}(\zeta)\right\}$, where $E_{4}(\zeta)$ is a sufficiently large constant, depending on the disorder $\zeta$, which will be fixed later on. For any subset $\Lambda \subset \mathbb{Z}^{v}$ and any finite interval $I \subset \mathbb{R}$, we introduce the set

$$
\begin{aligned}
\mathscr{B}_{k}(\Lambda, I)= & \left\{V: S_{k-1}(E, V) \cap \Lambda \neq \emptyset, \text { for some } E \in I, \text { and } \operatorname{dist}\left(\sigma\left(H_{C \cap A}^{0}\right), j^{2} E\right)\right. \\
& \left.\geq j^{2} e^{-d_{k-1}^{\gamma}}, \forall C \in \mathscr{C}, \forall j \in \mathbb{N}\right\} .
\end{aligned}
$$

Lemma 3.1. For $\gamma \in\left(0, \frac{1}{2}\right]$ sufficiently small, we have that

$$
\operatorname{Prob}\left(\mathscr{B}_{k}(\Lambda, I)\right) \leq|\Lambda| d_{k}^{-v-1} .
$$

The proof of Lemma 3.1 is similar to the one of Lemma 3.3 in [FMSS]. Moreover, by applying the first Borel-Cantelli lemma, as in the proof of Lemma 2.4 of $[\mathrm{AF}]$, one can prove the following result.

Lemma 3.2. For a.e. $V$, there exists an integer $k_{1}(V)<\infty$ such that

$$
S_{k}(E, V) \cap A_{k}=\emptyset,
$$

for all $k \geq k_{1}$ and all $E$ such that $\mathfrak{R} E \in \mathscr{G}\left(k_{1}, V\right)$, where the annuli $A_{k}$ are defined as in Sect. 2 after (2.5), and the gap sets $\mathscr{G}(k, V)$ are defined in (2.12).

We also require the following lemma.

Lemma 3.3. For almost every $V$ and for all eigenvalues $E_{0}$ of $H^{0}=-\Delta+V$ with $E_{0}>E_{4}(\zeta)$, for some constant $E_{4}(\zeta)$ that vanishes for large $\zeta$, there exists a finite integer $k_{2}\left(E_{0}, V\right)$ such that, for $\gamma \in\left(0, \frac{1}{2}\right]$ sufficiently small, and for all $k^{\prime}>k_{2}\left(E_{0}, V\right)$, the following statements hold:

(i) $\operatorname{dist}\left(\sigma\left(H_{C \cap \tilde{A}_{k^{\prime}}}^{0}\right), E_{0}\right)<e^{-d_{k^{\prime}-1}^{\gamma}}$

for some $C \in \mathscr{C}$;

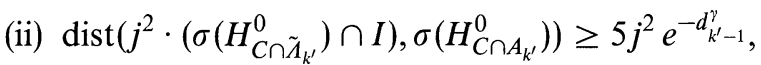

for all $j \in \mathbb{N}$, all $C, C^{\prime} \in \mathscr{C}$ and all finite intervals $I \subset \mathbb{R}$;

(iii) $\operatorname{dist}\left(j^{2} E_{0}, \sigma\left(H_{C \cap A_{k^{\prime}}}^{0}\right)\right) \geq 4 j^{2} e^{-d_{k^{\prime}-1}^{\gamma}}$

for all $j \in \mathbb{N}$;

(iv) $E_{0} \in \mathscr{G}\left(k_{2}, V\right)$.

Proof. Statement (i) is proven in Sect. 3 of [FMSS]. Statement (ii) is a generalization of (2.19) in [AF] and can be proven in the same way by combining the first Borel-Cantelli lemma and the Wegner estimate. Statement (iii) follows from (i) and (ii) and extends Lemma 2.5 of [AF]. Finally, (iv) follows from (iii) and definition (2.12) of the gap set $\mathscr{G}\left(k_{2}, V\right)$. It generalizes Corollary 2.6 of [AF].

Lemma 3.4. For a.e. $V$, and for all

$$
E_{0} \in \sigma(-\Delta+V) \cap\left\{E>E_{4}(\zeta)\right\},
$$

there exist a constant $\bar{k}\left(E_{0}, V\right)<\infty$ and a sequence $R_{n} \subset \mathbb{Z}^{v}$ of $(\bar{k}-2+n)$-admissible sets such that

(i) $\tilde{\Lambda}_{\bar{k}-2+n} \subset R_{n} \subset \Lambda_{\bar{k}-2+n}$ 
and

$$
\frac{1}{5} d_{\bar{k}-2+n} \leq \operatorname{dist}\left(\partial R_{n}, \partial \tilde{\Lambda}_{\bar{k}-2+n}\right) \leq \frac{2}{5} d_{\bar{k}-2+n},
$$

(ii) $\#\left\{E \in \sigma\left(H_{R_{n}}^{0}\right) \mid \operatorname{dist}\left(E, j^{2} E_{0}\right)<j^{2} e^{-\sqrt{d_{\bar{k}-3+n}}}\right\}= \begin{cases}1 & \text { for } j=1, \\ 0 & \text { for } j>1,\end{cases}$

(iii) $\operatorname{dist}\left(E_{0}, \sigma\left(H_{R_{n}}^{0}\right)\right) \leq e^{-\frac{1}{2} m d_{n}}$, for some constant $m>\frac{1}{2} m\left(E_{0}\right)$.

Proof. This lemma extends Lemma 2.8 of [AF]. In particular, statements (i) and (iii) can be inferred from this paper. The only claim that needs to be proven is (3.24), for $j>1$. Appealing to the first Borel-Cantelli lemma, and making use of the fast decay of the probability distribution of $V$, we see that there is a $k_{3}\left(E_{0}, V\right)<\infty$ such that, for all $k \geq k_{3}\left(E_{0}, V\right)$, we have that

$$
S_{0}^{(j)}\left(E_{0}, V\right) \cap \Lambda_{k}=\emptyset
$$

for all $j$ so large that

$$
j^{2} E_{0} \geq d_{k}
$$

Moreover, due to Lemma 3.2, it is also true that

$$
S_{k}^{(j)}\left(E_{0}, V\right) \cap A_{k}=\emptyset,
$$

for all $j \in \mathbb{N}$ and all $k \geq k_{1}(V)$. In particular, if $j^{2} E_{0} \geq d_{k_{4}}$, where $k_{4}:=$ $\max \left(k_{1}, k_{3}\right)$, then it follows that

$$
S_{k}^{(j)}\left(E_{0}, V\right) \cap \Lambda_{k}=\emptyset,
$$

for all $k \geq k_{4}$. Thanks to Lemma 7.3 of [AFS], we have that

$$
\operatorname{dist}\left(j^{2} E_{0}, \sigma\left(H_{R_{n}}^{0}\right)\right)>j^{2} e^{-\sqrt{d_{\bar{h}-3+n}}},
$$

for all $n$, where $\bar{k}=\max \left(k_{1}, k_{2}, k_{3}\right)$.

Hence (3.24) holds for all $j \in \mathbb{N}$ with the property that $j^{2} E_{0} \geq d_{k_{4}}$. i.e., (3.24) could fail only for $j \leq d_{k_{4}}^{1 / 2} E_{0}^{-1 / 2}$. However, if we choose $\bar{k}$ large enough this cannot happen. To see this, let us assume the contrary. Then there must be a $j_{0} \leq d_{k_{4}}^{1 / 2} E_{0}^{-1 / 2}$, and a subsequence $R_{n(l)}, l \in \mathbb{N}$, such that (3.28) fails for $j=j_{0}$ and all $l \in \mathbb{N}$. In this case, there must be a sequence

$$
u_{n(l)} \in l^{2}\left(R_{n(l)}\right)
$$

of eigenfunctions of $H_{R_{n(l)}}^{0}$ with eigenvalue $E_{n(l)}$ in the interval

$$
\left(j_{0}^{2} E_{0}-j_{0}^{2} e^{-\sqrt{d_{\bar{k}-3+n}}}, j_{0}^{2} E_{0}+j_{0}^{2} e^{-\sqrt{d_{\bar{k}-3+n}}}\right), \quad n \equiv n(l), \quad l=1,2,3, \ldots .
$$

By Lemmas 3.2 and 3.3 and the results of [FMSS], the eigenfunctions $u_{n(l)}$ have uniform exponential decay outside $\tilde{\Lambda}_{\bar{h}-2}$. Thus the $l^{2}$-limit, as $l \uparrow \infty$, of a subsequence of $u_{n(l)}$ exists and is an eigenfunction of $-\Delta+V$ on $l^{2}(\Lambda)$ with eigenvalue $j_{0}^{2} E_{0}$. But this is excluded in the hypotheses of Theorem 1 .

The next problem to be addressed concerns the modification of the definition of the singular sets $S_{k}^{j \delta v}(E, V)$ in [AF]. Let $v \in \mathscr{V}_{\bar{k}}$, where $\mathscr{V}_{\bar{k}}$ is defined in (2.7). Since $\tau=\left|\partial_{t}^{2}\right|^{-1 / 2}$ is a bounded operator from $L_{\text {odd }}^{2}(-\pi, \pi)$ to $L_{\text {odd }}^{\infty}(-\pi, \pi)$, and 
since $W(|y-x|)$ decays exponentially fast with rate $m_{W}>0$, as $|x-y| \uparrow \infty$, we have that, as a multiplication operator (in the time variable) on $L^{2}(-\pi, \pi)$,

$$
W(\tau v)(x, t):=\sum_{y} W(|y-x|)|(\tau v)(y, t)|^{2}
$$

is bounded in norm by const $\bar{k}_{\bar{k}} e^{-M|x|}$, where

$$
M=\min \left\{\frac{1}{2} m_{W}, \frac{1}{2} m\left(E_{0}\right)\right\} .
$$

Thanks to this norm-bound, the perturbation $\tau W(\tau v) \tau$ of $\tau(-\Delta+V) \tau$ is small relative to the sizes of local gaps in the spectrum of $\tau(-\Delta+V) \tau$. More precisely, we introduce singular sets $S_{k}^{\lambda \delta v}(E, V)$, as for the operator $\tau(-\Delta+V) \tau$, with the only modification that the spectral condition (3.6) is replaced by

$$
\operatorname{dist}\left(\sigma\left((-\Delta+V+(\lambda+i \delta) W(|\tau v|))_{\bar{C}_{k}^{\alpha}}\right), j^{2} \mathfrak{R} E\right) \geq \frac{1}{2} j^{2} e^{-\sqrt{d_{k}}}
$$

for all $j \in \mathbb{N}$. Lemma 2.9 of $[\mathrm{AF}]$ still holds with our new definition of singular sets, as the reader easily checks. The rest of the proof of [AF] can be extended to the present case with no essential modifications.

Remarks. (1) Writing out a self-contained proof of Theorem 1 would obviously be a rather lengthy enterprize. Since such a proof would repeat many arguments explained in detail in [AF] and in [FMSS], we decided to report, in the present paper, only those parts that contain some novel elements. (We realize that this makes the technical sections of our paper somewhat unreadable.)

(2) We expect to be able to construct solutions of the non-linear wave equation (1.1) which are quasi-periodic in the time variable $t$. More precisely, we expect it is possible to construct infinitely many finite-dimensional (and infinite-dimensional, compact) invariant tori in the phase space of the Hamiltonian system associated with Eq. (1.1), provided the non-linearity is kept small and the random function $V$ is chosen judiciously so as to escape from resonances. Those tori will be close to certain tori of the linear system $(\lambda=0)$.

Acknowledgements. We thank C. Eugene Wayne for some helpful discussions on problems related to the ones studied in this paper.

\section{References}

[AF] Albanese, C., Fröhlich, J.: Periodic Solutions ... I. Commun. Math. Phys. 116, 475 (1988)

[AFS] Albanese, C., Fröhlich, J., Spencer, T.: Periodic Solutions ... II. Commun. Math. Phys. 119, 677 (1988)

[FMSS] Fröhlich, J., Martinelli, F., Scoppola, E., Spencer, T.: Constructive proof of localization in the Anderson tight binding model. Commun. Math. Phys. 101, 21 (1985)

[FS] Fröhlich, J., Spencer, T.: Absence of diffusion in the Anderson tight binding model for large disorder or low energy. Commun. Math. Phys. 88, 151 (1983)

[SW] Simon, B., Wolff, T.: Singular continuous spectrum under rank one perturbations and localization for random Hamiltonians. Commun. Pure Appl. Math. 39, 75 (1985)

[DLS] Delyon, F., Lévy, Y., Souillard, B.: Anderson localization for multidimensional systems at large disorder or low energy. Commun. Math. Phys. 100, 463 (1985)

[DK] von Dreyfus, H., Klein, A.: Irvine preprint (1988) 
